These criteria allow for local variation (e.g. whether Korsakoff's syndrome is included in criterion 1) and are based on judgement rather than reductionism. Consequently, they may lead to disagreements about individual patients which are probably best addressed through conjoint management. On the whole, however, they are seen as logical and utilitarian. Modelling suggests workload of old age services would be similar to now if they are implemented.

I agree with Dr Sikdar that it is really important to preserve management of functional illnesses in old age teams. Almost $50 \%$ of my directorate's workload is non-organic and the crossover between dementia and functional illness is very common and complex. Many people with dementia present with functional symptoms and many more will develop severe functional symptoms once dementia is established (psychosis $25 \%$, depression 40\%). Many people with long-established functional illness will develop dementia. For these reasons, the only way it is possible to preserve continuity of care is to have old age teams providing care across the spectrum.

The disproportionate funding gap (old age services are underfunded by over $€ 2$ billion compared with adult services) is an outrage, especially as we face real growth in demand owing to demographic change. The fact that trusts' cost improvement plans are equally applied to old age services is unconscionable and illogical; but would be up to local clinicians to argue this point.

The Faculty has done much over the past few months to address these issues, for example through a national survey of our members to establish baseline service provision and map changes, lobbying politicians and trusts and development of new service criteria. There is much still to do and the support of our members in this work remains pivotal.

James Warner, Chair, Old Age Faculty, Royal College of Psychiatrists, London, UK, email: j.warner@imperial.ac.uk

doi: $10.1192 / p b .37 .3 .116 a$

\section{Trainees want to work out of hours!}

We welcome Oakley et al's timely contribution to the debate around the future of psychiatric training. ${ }^{1}$ The issue of decreasing exposure to emergency assessments out of hours has been of concern to the Psychiatric Trainees' Committee for several years. ${ }^{2}$ Originally a fear prompted by the introduction of working time regulations, our unease is now focused on the impact of widespread service reconfigurations; in some areas these are resulting in rotas which dramatically restrict trainees' exposure to emergency learning experiences.

Are presentations qualitatively different out of hours? Research in this regard is limited, but it is our unanimous opinion that outside of 9am to $5 \mathrm{pm}$ working hours and particularly at night, patients tend to present with more complex and challenging problems, often involving dual diagnoses, drug and alcohol intoxication and higher rates of self-harm and overdose. Services available are also more limited, making decisions about care more demanding.

We concede that out-of-hours rotas which involve trainees working off site, or not at all, may increase the trainee's time spent with patients in day hours, providing continuity of care and the opportunity to attend teaching and academic programmes. However, daytime work is often centred on service provision and routine tasks, rather than acquiring essential emergency competencies required to be a successful and accountable higher trainee and consultant.

Decreasing trainees' work out of hours may also serve to further devalue the image of psychiatry to other medical professionals - when seeking advice on a complex issue of risk, capacity or consent, for example, they expect to be able to speak to a medically qualified professional. As is well recognised, improving the image of psychiatry as a specialty is key to reducing professional stigma and promoting recruitment.

In this same edition of The Psychiatrist, Tadros et al describe a revolutionary and highly successful 24-hour, 7-daysa-week method of working. ${ }^{2}$ We are of the opinion that the more services are designed with well-supported core trainees working at the coalface, particularly out of hours, the more training standards will improve and the more our specialty will be valued within the multidisciplinary environment.

We also believe that having medically trained professionals on the frontline makes for safer services.

The Psychiatric Trainees' Committee has established a working group to look into the provision of out-of-hours services across the UK, with a particular aim of understanding how training might be affected by reductions in out-of-hours experience. We will present the findings to the College and hope to work towards a shared understanding of the future of out-of-hours training.

Submitted on behalf of the Psychiatric Trainees' Committee.

1 Oakley C, Jenkinson J, Oyebode F. Psychiatric training for the next generation. Psychiatrist 2013; 37: 25-9.

2 Royal College of Psychiatrists' Psychiatric Trainees Committee. Finding the Balance: The Psychiatric Training Value of Out of Hours Working. Royal College of Psychiatrists 2008 (www.rcpsych.ac.uk/ pdf/PTC\%20The\%20training\%20value\%20of\%200OH.pdf).

3 Tadros G, Salama RA, Kingston P, Mustafa N, Johnson E, Pannell R, et al Impact of an integrated rapid response psychiatric liaison team on quality improvement and cost savings: the Birmingham RAID model. Psychiatrist 2013; 37: 4-10.

Rory Conn, CT3 Psychiatry, Camden and Islington NHS Foundation Trust, Out-Of-Hours Working Group Lead, Royal College of Psychiatrists' Psychiatric Trainees' Committee, London, email: rconn@doctors.org.uk, Muj Husain, Clinical Fellow, General Medical Council, NHS Medical Director's Scheme, Chair, Royal College of Psychiatrists' Psychiatric Trainees' Committee.

doi: $10.1192 / \mathrm{pb} .37 .3 .117$ 